\title{
Broadening of Tunable Range of Resonant Frequency of Cylindrical Cavity Resonator of a Particular Mode Avoiding Mode Overlapping
}

\author{
Prasun Banerjee ${ }^{1}$, Sanjay Mondal' ${ }^{2}$, Salil Kumar Biswas ${ }^{3}$ \\ ${ }^{1}$ Instituto de Física, Universidade Federal De Goiás, Goiânia, Goiás, Brasil \\ ${ }^{2,3}$ Department of Physics, University College of Science and Technology, University of Calcutta, Kolkata, India
}

\begin{abstract}
The tuning of microwave cavity resonator is very important technique to improvise selective component in the field of microwave technology. Tuning is done by various ways. Among those one of the most convenient ways of tuning is done by variation of the dimension of the cavity. During the process of tuning the resonant frequency of different modes moves differently. As a result overlapping of resonant frequencies of different modes may occur. This causes noise or other problem. Here we describe a technique to sort out that problem. Here we also describe the method how to increase the frequency gap between successive resonant frequencies of different modes of cylindrical cavity resonator.
\end{abstract}

Keywords: Microwave cavity resonator, resonance mode, tuning, resonant frequency

\section{INTRODUCTION}

In general, the cavity resonators that confine the electromagnetic energy are simply waveguides of specific dimension short circuited at both ends. Nowadays electromagnetic cavity has been one of the most important tools and their applications are vast and range from the use as frequency meters and filters, to tools for material characteristics measurements [1-12]. Tuning of this cavity at various frequencies is necessary for those purposes. We may vary the height of a cylindrical cavity resonator by moving one plane side wall of the cavity for our tuning purpose, because of the dependency of resonant frequency on the height of the resonator. But this variation is not same for all the modes exist in the cavity. Particularly the resonant frequency of $\mathrm{TM}_{\mathrm{mn} 0}$ mode has no variation with

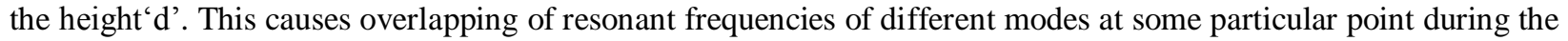
variation of height which may cause noise problem. To overcome this problem we use a wall with curvature, as our moving wall, to vary the height of the cavity. As a result of this curvature the resonant frequencies of different modes move differently from the point of overlapping and we can tune a particular mode at that point. If we can increase the frequency gaps between two successive modes then we can vary our desired resonant frequency to a greater extent without overlapping with the other modes. The increments of the frequency gap between the successive modes can be done by the coating of dielectric material like silicon di oxide on inner curved wall of the cylindrical cavity.

\section{Theoretical Analysis}

The oretical analysis of cylindrical cavity is quite complex [13]. To analyze the field a simple field model is required. The electromagnetic field of a circular cylindrical resonator can be written as [14]

$$
\begin{aligned}
& H_{z}=H_{0} J_{1}\left(\frac{p_{11}^{\prime} \rho}{a}\right) \cos \phi \sin \frac{l \pi z}{d} \\
& H_{\rho}=\frac{H_{0} \beta a}{p_{11}^{\prime}} J_{1}^{\prime}\left(\frac{p_{11}^{\prime} \rho}{a}\right) \cos \phi \cos \frac{l \pi z}{d} \\
& H_{\phi}=\frac{-\beta a^{2} H_{0}}{\left(p_{11}^{\prime}\right)^{2} \rho} J_{1}\left(\frac{p_{11}^{\prime} \rho}{a}\right) \sin \phi \cos \frac{l \pi z}{d}
\end{aligned}
$$

\footnotetext{
${ }^{1}$ Corresponding Author: prasun@ufg.br
} 


$$
\begin{aligned}
& E_{\rho}=\frac{j k \eta a^{2} H_{0}}{\left(p_{11}^{\prime}\right)^{2} \rho} J_{1}\left(\frac{p_{11}^{\prime} \rho}{a}\right) \sin \phi \sin \frac{l \pi z}{d} \\
& E_{\phi}=\frac{j k \eta a H_{0}}{\left(p_{11}^{\prime}\right)^{2} \rho} J_{1}^{\prime}\left(\frac{p_{11}^{\prime} \rho}{a}\right) \cos \phi \sin \frac{l \pi z}{d} \\
& E_{z}=0
\end{aligned}
$$

Where $\mathrm{d}$ is the cavity height, a is the radius, $k=\omega \sqrt{\mu \varepsilon}, \eta=\sqrt{\frac{\mu}{\varepsilon}}$ and $J_{1}\left(\frac{p^{\prime} \rho}{a}\right)$ denote the Bessel functions of order one.

The unloaded quality factor of the cavity can be expressed as

$$
Q=\omega \frac{W_{M}+W_{E}}{P_{D}+P_{C}}
$$

Where $\mathrm{W}_{\mathrm{M}}$ and $\mathrm{W}_{\mathrm{E}}$ are the time average of stored magnetic and electric energies, $\mathrm{P}_{\mathrm{D}}$ and $\mathrm{P}_{\mathrm{C}}$ are the dissipated power in the dielectric and conducting wall, respectively. At the resonance $\mathrm{W}_{\mathrm{M}}=\mathrm{W}_{\mathrm{E}}$, hence

$$
Q=\omega \frac{2 W_{M}}{P_{l o s s}}
$$

Where $\mathrm{P}_{\text {loss }}=\mathrm{P}_{\mathrm{D}}+\mathrm{P}_{\mathrm{C}}$ is the total power dissipated.

At resonance total energy is

$$
W=2 W_{E}=\frac{\varepsilon}{2} \int_{z=0}^{d} \int_{\phi=0}^{2 \pi} \int_{\rho=0}^{a}\left(\left|E_{\rho}\right|^{2}+\left|E_{\phi}\right|^{2}\right) \rho d \rho d \phi d z
$$

The power loss due to the conducting wall is

$$
P_{C}=\frac{R_{S}}{2} \int_{S}\left|\bar{H}_{\mathrm{tan}}\right|^{2}
$$

The quality factor with imperfectly conducting walls is

$$
Q=\omega \frac{W}{P_{C}}
$$

The power dissipated in the dielectric can be calculated as

$$
P_{D}=\frac{1}{2} \int_{V} \bar{J} \cdot E^{*}
$$

Hence the quality factor is

$$
Q_{D}=\frac{\omega W}{P_{D}}
$$

So total quality factor $\mathrm{Q}$

$$
Q=\left(\frac{1}{Q_{C}}+\frac{1}{Q_{D}}\right)^{-1}
$$


Hence the resonant frequency is

$$
\left(f_{r}\right)_{m n l}=\frac{1}{2 \pi \sqrt{\mu \varepsilon}} \sqrt{\left(\frac{p_{m n}}{a}\right)^{2}+\left(\frac{l \pi}{d}\right)^{2}}
$$

\section{Experimental Details}

It is obvious from equation (15) that the resonant frequency is related to the dimension of the cavity. Variation of resonant frequency, $\mathrm{f}_{\mathrm{r}}$ with the height ' $\mathrm{d}$ ' of the cavity for $\mathrm{TM}_{110}$ mode and $\mathrm{TM}_{011}$ mode is shown in figure 1 .

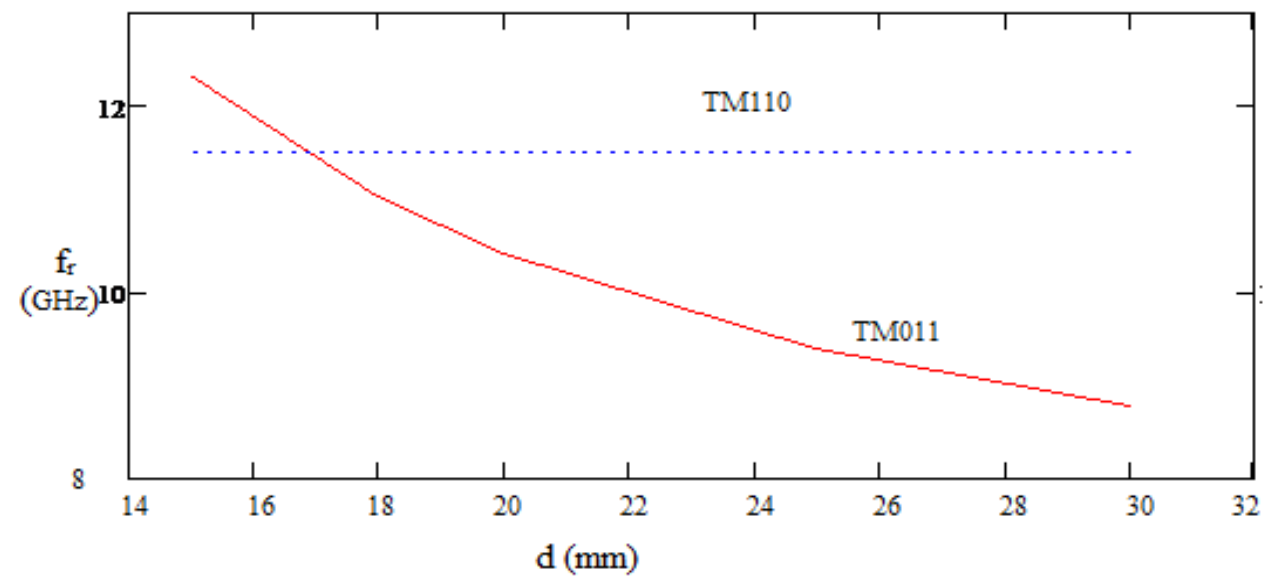

Fig1. Variation of resonant frequency with height 'd'(from eqn. 15)

From figure 1 , the resonant frequency of $\mathrm{TM}_{110}$ mode and $\mathrm{TM}_{011}$ mode coincide at the frequency $11.51 \mathrm{GHz}$ and at height $16.9 \mathrm{~mm}$. Our aim is to isolate that resonant frequency of $\mathrm{TM}_{011}$ mode from $\mathrm{TM}_{110}$ mode.

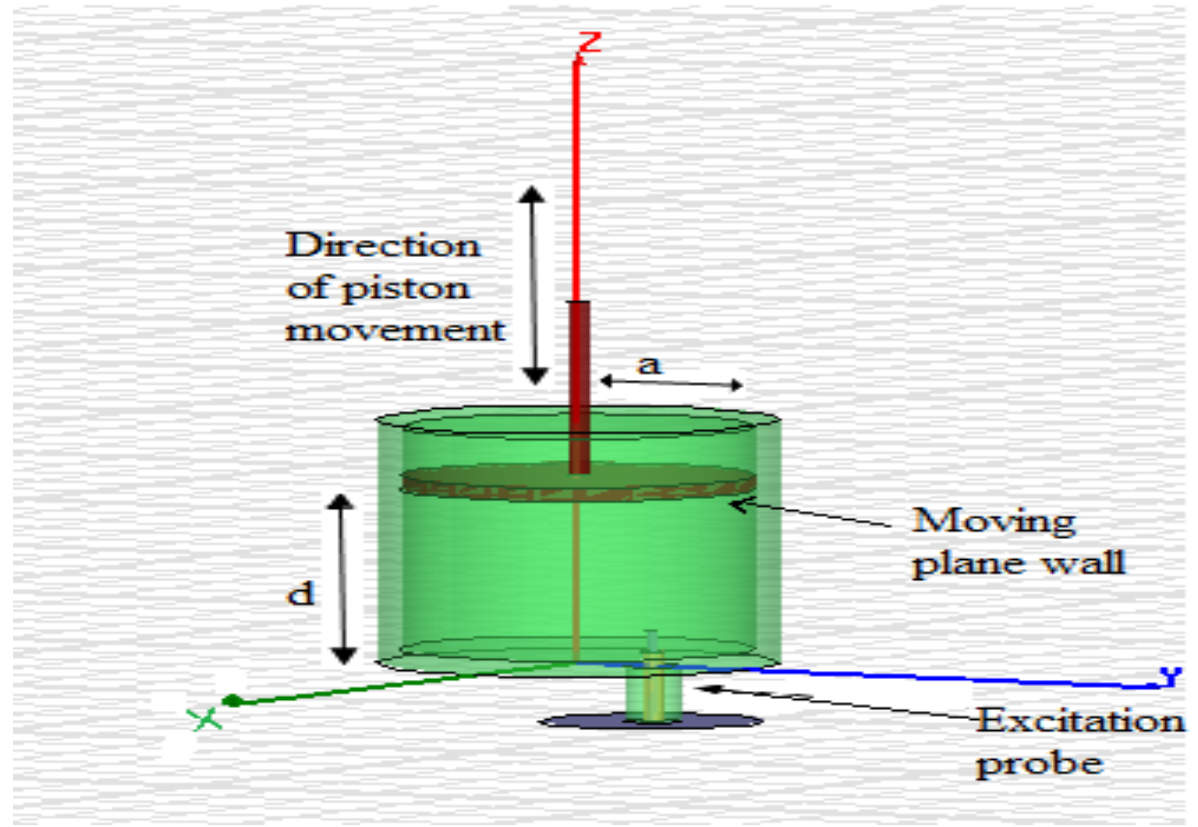

Fig2. Cavity with moving wall to the direction of Z-axis.(Designed in HFSS)

At first, the cylindrical cavity resonator simulated in the HFSS for $\mathrm{a}=15.9 \mathrm{~mm}$ and $\mathrm{d}=30 \mathrm{~mm}$. Figure 3 gives the measured reflection coefficient from the cavity with plane moving wall at different frequencies from which we can calculate resonant frequencies of different mode. 
American Research Journal of Physics, Volume 1, Issue 1, 2015

ISSN 2380-5714

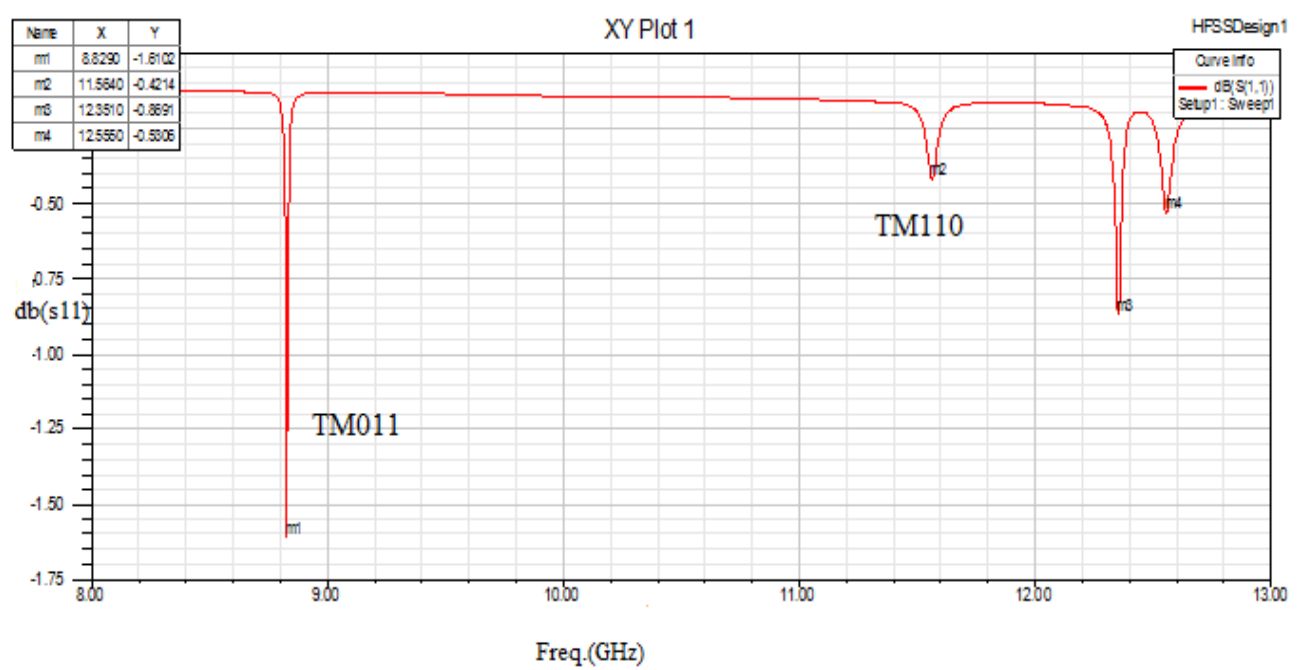

Fig3. $S_{11}$ characteristics obtained from the simulation of the cavity with plane moving wall

Table1. The table given below shows a well agreement of simulated values with the theoretical values

\begin{tabular}{|l|c|c|}
\hline MODE & Theoretical value of resonant frequency in $\mathrm{GHz}$ & Simulated value of resonant frequency in $\mathrm{GHz}$ \\
\hline $\mathrm{TM}_{011}$ & 8.79 & 8.82 \\
\hline $\mathrm{TM}_{012}$ & 11.51 & 11.56 \\
\hline
\end{tabular}

Now we observe effect of curvature on the plane moving wall. We use the curvature with $4 \mathrm{~mm}$ deep from the plane moving wall. The design of that cavity which will be simulated for its different height in HFSS is shown in fig.4.

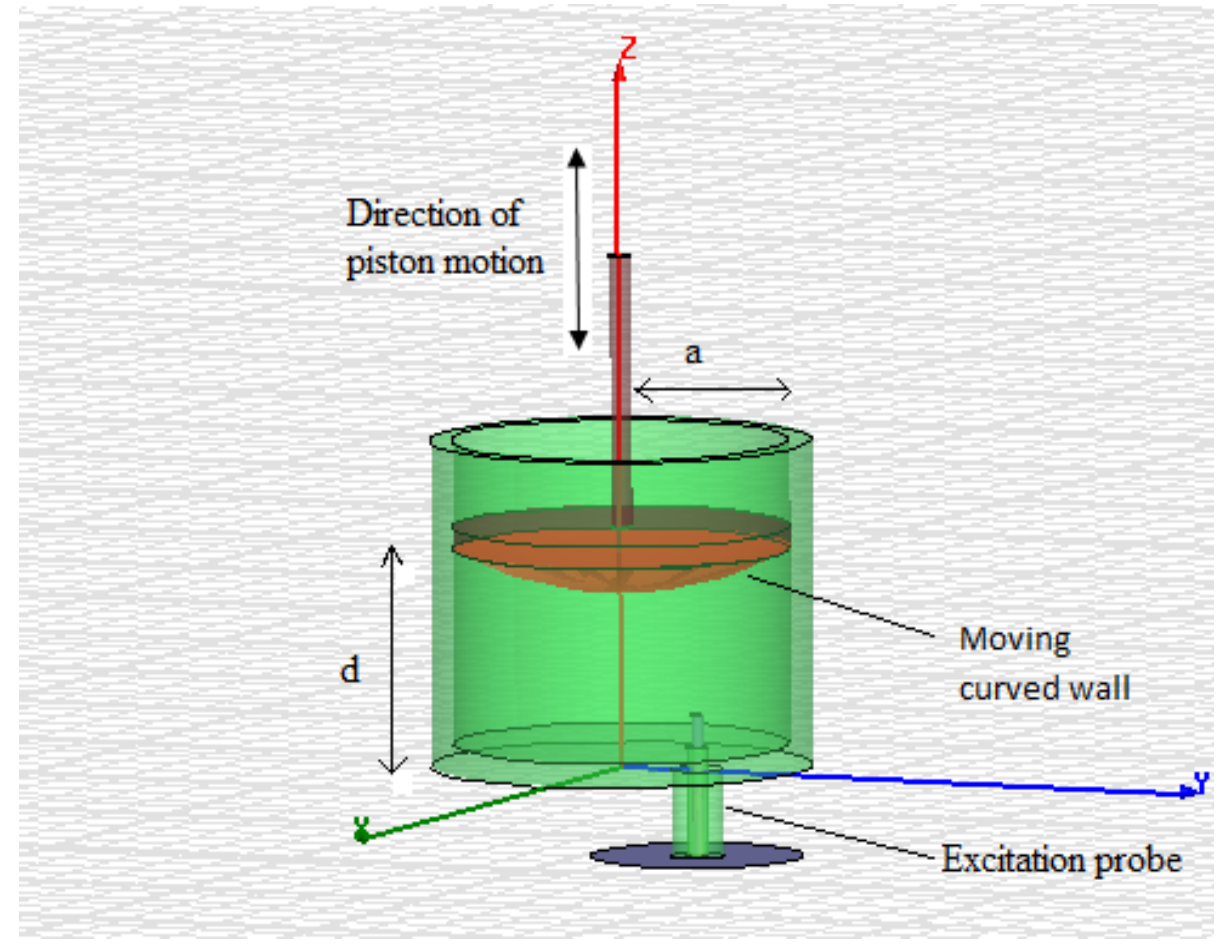

Fig4. Cavity with moving wall with curvature to the direction of Z-axis. (Designed in HFSS)

Figure 5 gives the measured reflection coefficient from the cavity with moving wall with $4 \mathrm{~mm}$ curvature at different frequencies for $\mathrm{a}=15.9 \mathrm{~mm}$ and $\mathrm{d}=30 \mathrm{~mm}$ from which we can calculate resonant frequencies of different mode. 
American Research Journal of Physics, Volume 1, Issue 1, 2015

ISSN 2380-5714

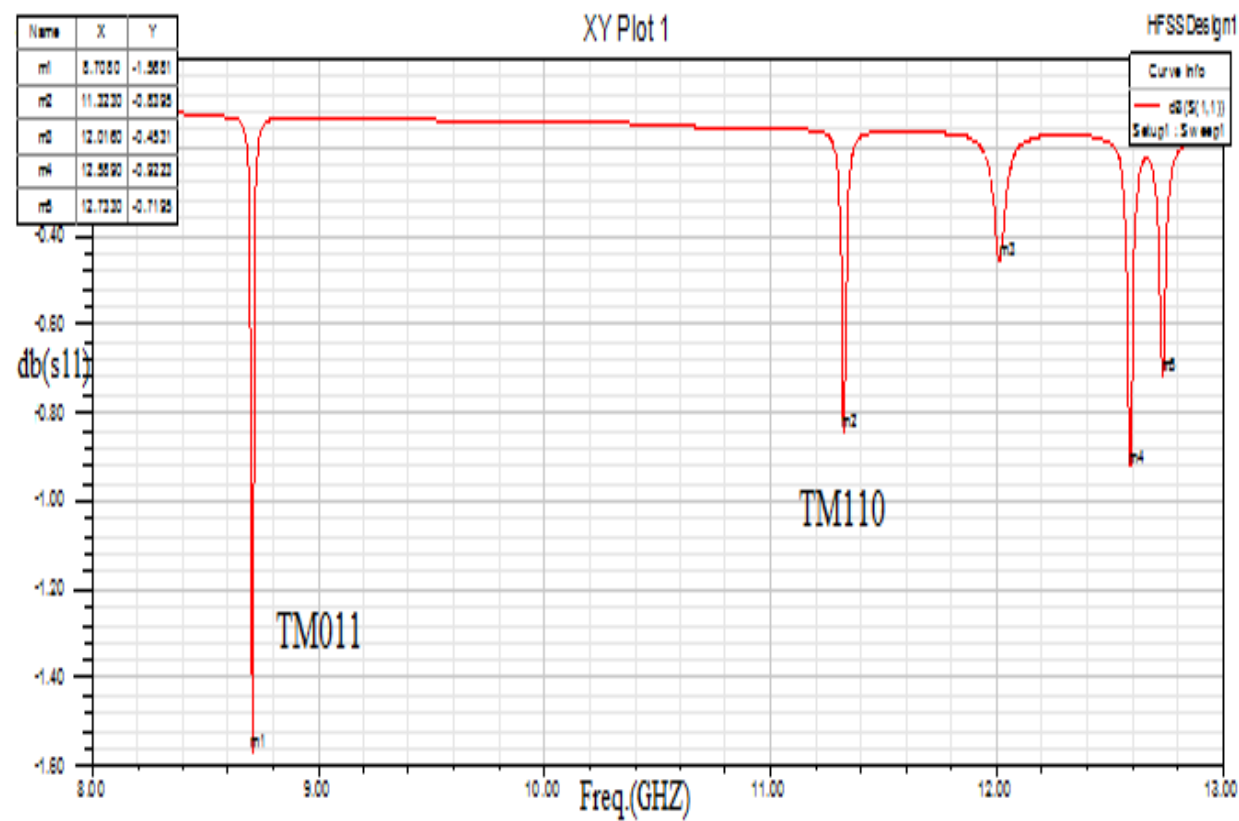

Fig5. S11 characteristics obtained from the simulation of the cavity with curved moving wall

From figure 5 it is seen that the resonant frequency of $\mathrm{TM}_{011}$ shifts from $8.82 \mathrm{GHz}$ to $8.71 \mathrm{GHz}$ and $\mathrm{TM}_{110}$ shifts from $11.56 \mathrm{GHz}$ to 11.32 . For both of the modes resonant frequencies decrease due to curvature. But those shifts are different. $\mathrm{TM}_{011}$ mode shifts $0.11 \mathrm{GHz}$, whereas $\mathrm{TM}_{110}$ mode shifts $0.24 \mathrm{GHz}$ for $4 \mathrm{~mm}$ curvature. Now we simulate the cavity with curved moving wall for different height and note the corresponding resonant frequency for each mode. The variation of resonant frequency with the height' $d$ ' for both of the mode is shown in figure 6 .

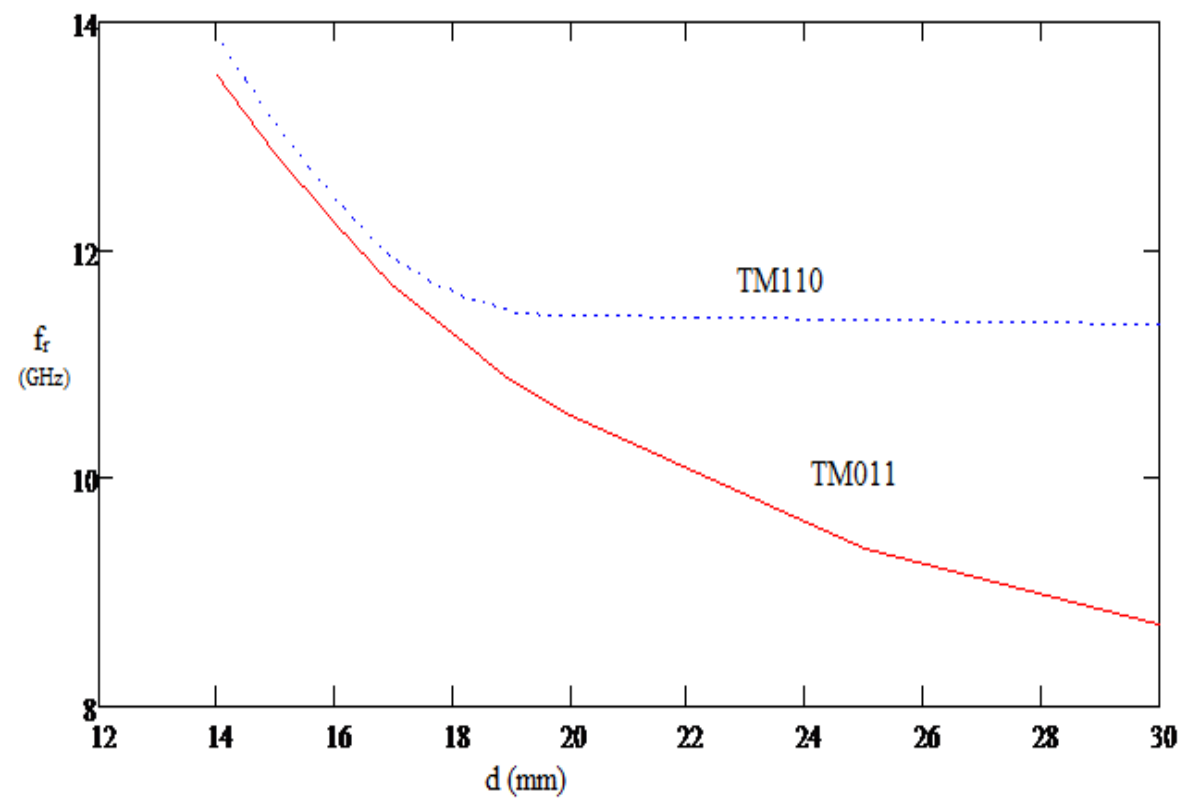

Fig6. Variation of resonant frequency of $T M_{011}$ and $T M_{110}$ mode with cavity height of a cylindrical resonator with curved moving wall obtained from HFSS simulation.

Figure 6 shows no intersection between the two curves in contrast with the figure 1. The constancy of resonant frequency of $\mathrm{TM}_{110}$ mode breaks up for the lower height of the cavity. Resonant frequency of $\mathrm{TM}_{011}$ mode without curvature was $11.51 \mathrm{GHz}$ (at the point of intersection with $\mathrm{TM}_{110}$ mode) for the height $16.9 \mathrm{~mm}$, whereas that 


\section{American Research Journal of Physics, Volume 1, Issue 1, 2015}

ISSN 2380-5714

resonant frequency occurs at the height $17.34 \mathrm{~mm}$ with the curvature at the moving wall and at this point the resonant frequency of $\mathrm{TM}_{110}$ is $11.82 \mathrm{GHZ}$. So the resonant frequency of $\mathrm{TM}_{110}$ mode shifts by an amount $0.31 \mathrm{GHz}$ from the point of intersection.

Now to understand the effect of coating in the inner curved wall we simulated the empty cavity with height $\mathrm{d}=4$ c.m. and radius a $=1.25 \mathrm{c} . \mathrm{m}$ and plotted the $\mathrm{S}_{11}$ curve. These results are then compared with the theoretical data.

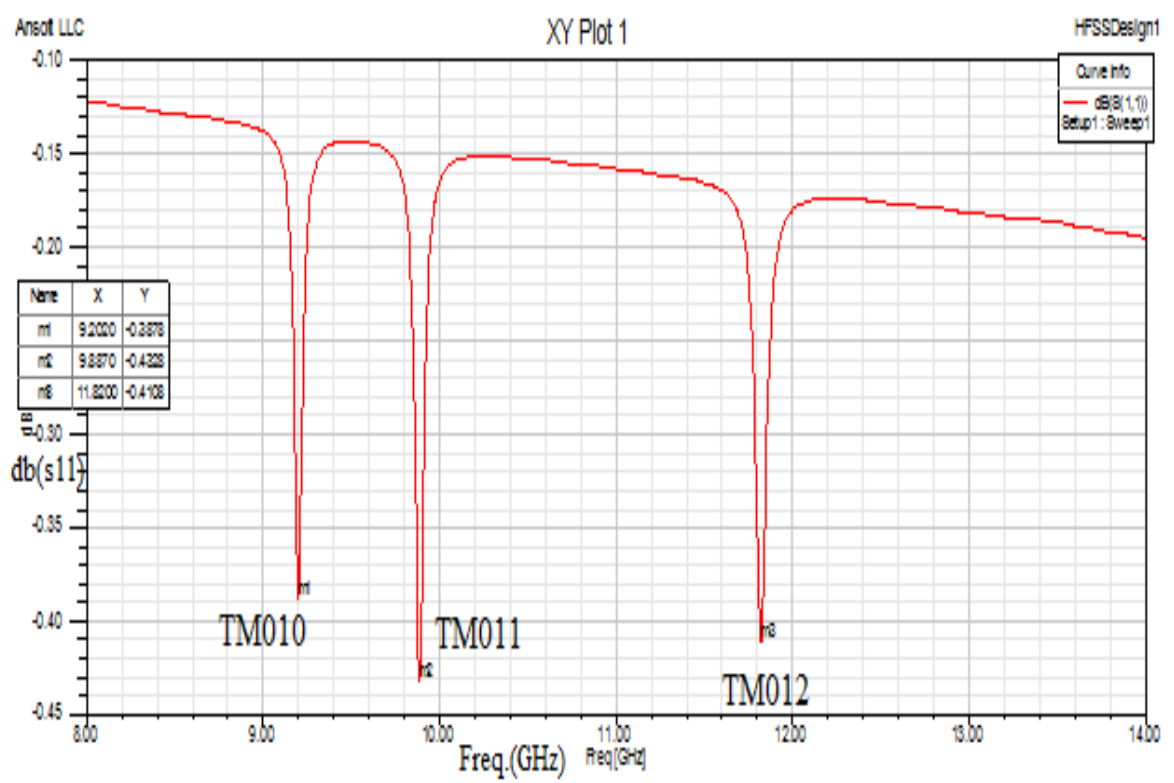

Fig.7

\begin{tabular}{|l|c|c|}
\hline MODE & Theoretical value of resonant frequency in $\mathrm{GHz}$ & Simulated value of resonant frequency in $\mathrm{GHz}$ \\
\hline $\mathrm{TM}_{010}$ & 9.191 & 9.202 \\
\hline $\mathrm{TM}_{011}$ & 9.927 & 9.887 \\
\hline $\mathrm{TM}_{012}$ & 11.860 & 11.820 \\
\hline
\end{tabular}

Now if we change the height of the cavity with the help of a moving plunger, then resonant frequencies of different mode except $\mathrm{TM}_{\mathrm{mn} 0}$ mode will change. But these changes would not be same for all modes. So overlapping of different modes may occur or they get closer to each other which may create problem of tuning.

This problem can be resolved to some extent if we increase the frequency gap between the resonant frequencies of successive modes. That can be done using a coating of dielectric material like $\mathrm{SiO}_{2}$ on the inner curved wall of the cavity. The $\mathrm{S}_{11}$ curve is given below.

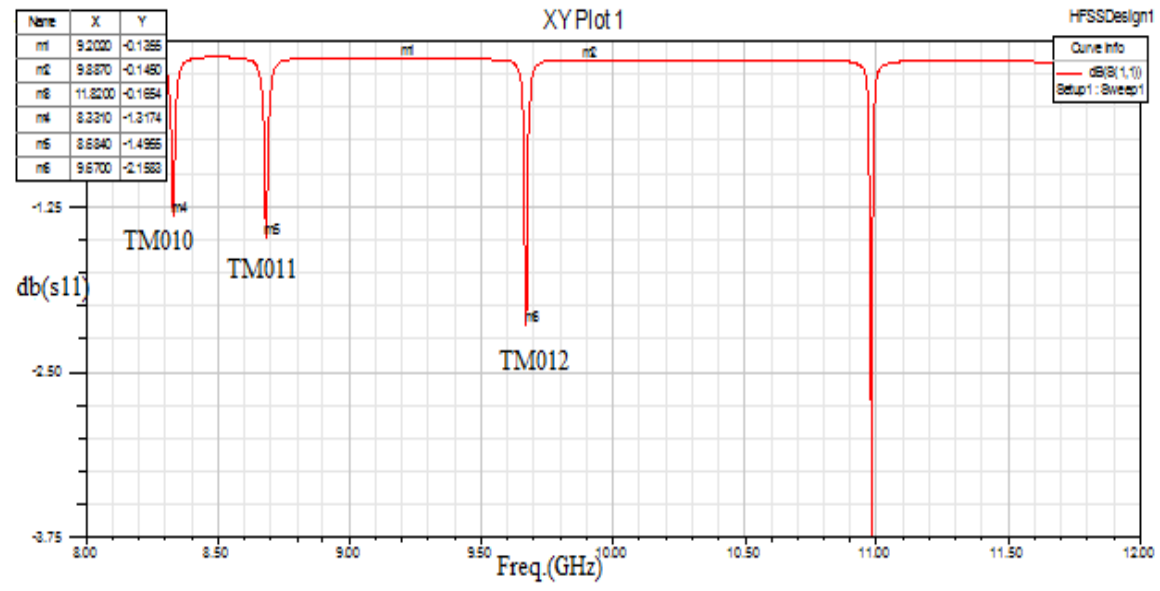

Fig. 8 
In figure 7 resonant frequencies for $\mathrm{TM}_{010}, \mathrm{TM}_{011}$ and $\mathrm{TM}_{012}$ are $9.202 \mathrm{GHz}, 9.887 \mathrm{GHz}$ and $11.820 \mathrm{GHz}$ respectively for the cavity with radius $12.5 \mathrm{~mm}$ and height $40 \mathrm{~mm}$ without coating. With $3 \mathrm{~mm} \mathrm{SiO}_{2}$ (dielectric constant $=4$ ) all the resonant frequencies of the respective mode decrease (Fig.8). To retain the resonant frequency of TM011 mode unchanged height of the cavity is reduced to $1.98 \mathrm{~cm}$ (Fig.9).

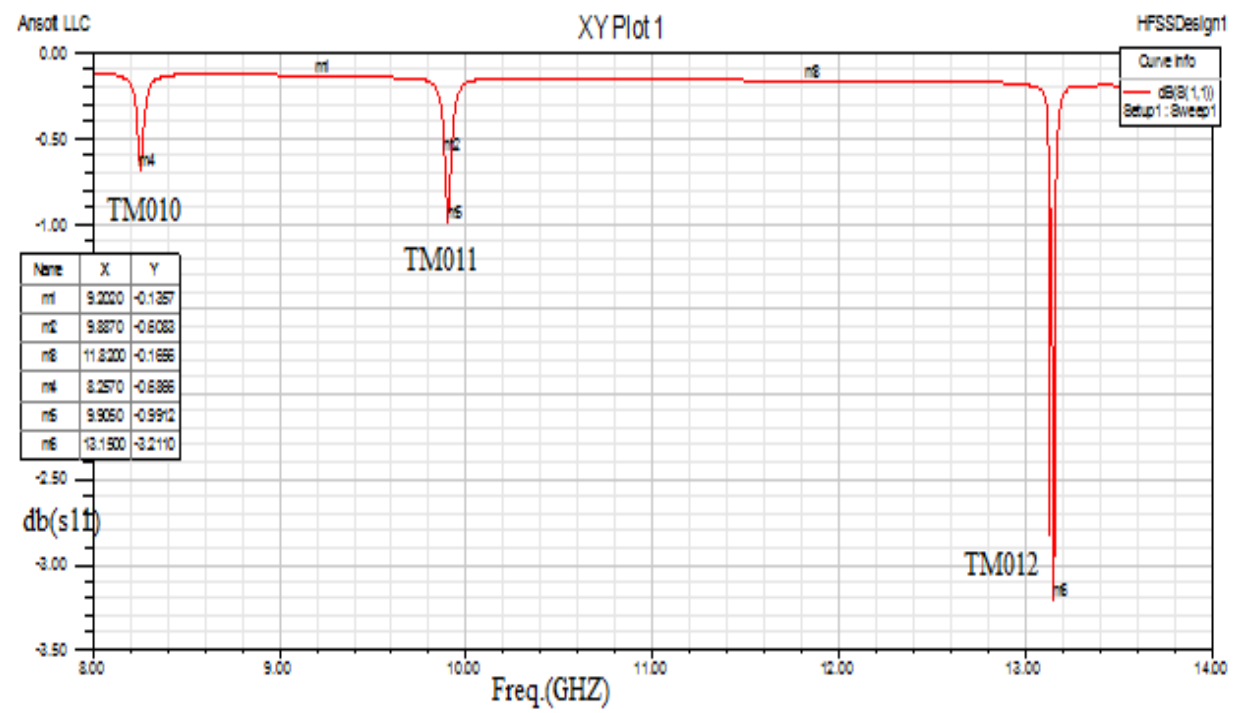

Fig. 9

As a result, the separation between $\mathrm{TM}_{010}$ and $\mathrm{TM}_{011}$ mode increased by an amount $0.963 \mathrm{GHz}$ and separation between $\mathrm{TM}_{011}$ and $\mathrm{TM}_{012}$ mode increased by an amount $1.312 \mathrm{GHz}$.

\section{CONCLUSION}

Separation of resonant frequency of different mode from their point of intersection can be done by using moving curved wall. But more work to be done to optimize the curvature for better separation. However Q-value of the cavity may decrease due to increment of surface area of curved moving wall. A study on this matter is necessary in future work. The frequency gaps between the resonant frequencies of successive modes for a particular range of frequency of a cavity resonator can be increased with the help of dielectric coating on the inner curved wall of the cylindrical cavity. As a result of that the tunable range of frequency for a particular mode may be increased without overlapping of resonant frequencies of different modes. However, further works are necessary to examine the variation of increments of tunable range with the amount of coating.

\section{REFERENCES}

[1] R.A.Desai et all, Computer Modeling of Microwave Cooking using the Transmission-line Model, IEE Proceedings, Pt.A, Vol.139, No.1, pp.30-37, 1992.

[2] M.Mladenovic, A.Marincic, B.Milovanovic, Mode Tuning of Microwave Resonator Loaded with Lossy Multilayer Dielectric, Journal of Microwave Power and Electromagnetic Energy, Vol.24, No.3, pp.166-177, 1989.

[3] T. Itoh, "A new method for Measuring Properties of dielectric Materials Using a Microstrip Cavity",(Short Paper), IEEE Trans. on microwave theory and technique, May 1974.

[4] M.Mladenovic, A.Marincic, B.Milovanovic, Calculation of Resonances of Microwave Resonator Loaded with Inhomogeneous Dielectric Layer, Electronics Letters, Vol.27, No.24, pp.2215-2216, 1991.

[5] A.Baysar, J.L.Kuester and S.El-Ghazaly, Theoretical and Experimental Investigations of Resonance Frequencies in a Microwave Heated Fluidized Bed Reactor, IEEE MTT-S Digest, pp.1573-1576, 1992.

[6] B.Milovanovic, S.Ivkovic, N.Doncov, D.Djordjevic, Resonant Frequencies Determination of Circular Cylindrical Cavities Loaded By One Lossy Dielectric Slab, Yugoslav IEEE MTT Chapter Informer, No.4, pp.33-37, 1996. 
[7] B.Milovanovic, N.Doncov, A.Atanaskovic, Tunnel Type Microwave Applicator Modelling using TLM Method, Problems in Modern Applied Mathematics: A Series of Reference Books and Textbooks: Mathematics and Computers in Science and Engineering, WSES Press, pp.327-332, 2000.

[8] B.Milovanovic, S.Ivkovic, N.Doncov, D.Djordjevic, Mode Tuning of Multilayer Cylindrical Cavities with Circular and Elliptical Cross-section, Journal of Microwave Power and Electromagnetic Energy, Vol.33, No.1, pp.49-56, 1998. Network, Proc. of the 5 th IEEE TELSIKS'01 Conference, Nis, Yugoslavia, pp.687-690, 2001.

[9] B.Milovanovic, V.Markovic, Z.Marinkovic, Z.Stankovic, Some Applications of Neural Networks in Microwave Modeling, Journal of Automatic Control, University of Belgrade, Yugoslavia, Vol.13(1), pp.39-46, 2003.

[10] K.S.Kunz, R.J.Luebbers, The Finite Difference Time Domain for Electromagnetics, CRC Press, 1993.

[11] C.Christopoulos, The Transmission-Line Modelling Method, IEE/OUP Press, 1995.

[12] Mohd Tarmizi Ali, Mohd Khairul Mohd Salleh, and Md. Mahfudz Md. Zan, Air-Filled Circular Cross Sectional Cavity for Microwave Non-Destructive Testing, Proceedings Of World Academy Of Science, Engineering And Technology Volume 18 December 2006 Issn 1307-6884

[13] P. Guillon and Y. Garault, Accurate resonant frequencies of dielectric resonators, IEEE Trans. Microwave Theory Tech.,vol.MTT-25, pp.916-922, Nov.1977

[14] David M. Pozar, Microwave Engineering, John Wiley \&Sons, inc.1988 